\title{
An Application of Augmented Reality to Electrical Power Equipment Maintenance
}

\author{
Jiang Zhu, Tao Yan, Chong Xie \\ NARI Group Corporation (State Grid Electric Power Research Institute) \\ Nanjing, Jiangsu, 210003, china \\ E-mail: zhujiang@sgepri.sgcc.com.cn
}

\begin{abstract}
With the development of science and technology in the power industry, the maintenance staffs of all power companies are facing more and more electrical power equipment. The internal structure of equipment is more complex. So are the external physical and logical relations of it. The traditional maintenance management system usually offers ways such as object tree, keyword search and chart zooming to find an item of equipment or a service node; and offer ways of the traditional spreadsheets and two dimension graphics to show the data. When dealing with complex business logic, the system often needs to use a lot of layered two dimension graphics. And users are difficult to get the key information at first. Users need to spend a lot of energy to remember and understand the relationship between the graphics, prone to miscarriage of justice. To overcome these difficulties, we developed an augmented reality-based power equipment maintenance system. A head-mounted display is used to observe and choose the specific power equipment. Needed data from information system are superimposed on the video image of the equipment. The business of the equipment is handled by data glove, the control key, gesture, voice, eye tracking, analog touch etc. The field experiments showed that our system could greatly reduce the workload of the maintenance staffs and improve work efficiency.
\end{abstract}

Keywords-ar; maintenance; power equipment; image recognition component

\section{INTRODUCTION}

Power equipment maintenance is very important for maintaining reliability of the equipment and extending the life of it. With the development of science and technology in the power industry, the maintenance staffs of all power companies are facing more and more electrical power equipment. The internal structure of equipment is more complex. So are the external physical and logical relations of it. The traditional maintenance management system usually offers ways such as object tree, keyword search and chart zooming to find an item of equipment or a service node; and offer ways of the traditional spreadsheets and two dimension graphics to show the data. When dealing with complex business logic, the system often needs to use a lot of layered two dimension graphics. And users are difficult to get the key information at first. Users need to spend a lot of energy to remember and understand the relationship between the graphics, prone to miscarriage of justice.

On the other hand, the quality of the on-site maintenance which can be categorized into many classes [1-2] is reflective of the skill of the maintenance technicians, their workmanship, and quality of the supporting documentation, procedures, and the technologies used. Portable computer and personal digital assistant (PDA) are helpful to it. But portable computer is usually too large for the working site; and PDA is often difficult for complex business. Furthermore, they are all inefficient because the maintenance staffs must frequently alternate their gaze between the working field and the laptop or PDA monitor.

A new method for electrical power equipment maintenance is required to improve work efficiency and reduce the workload of the maintenance staffs. In recent years, the mobile Internet is finally becoming a reality thanks to high-performance, low-power processing chips and effective high speed wireless networks. With the rapid development of mobile Internet, smart glasses, intelligent helmets have a swift and violent development. And based on video processing, AR and virtual reality (VR) technology, Microsoft, Google, EPSON and other well-known companies have launched their new products. All of these developments make the new method feasible in technology. In fact, we have invented a new electrical power equipment maintenance system that enables staffs to see needed information overlaid on the equipment. We have named it the Perspective Equipment Maintenance System (PEMS); PEMS fully adopts the AR technique.

\section{SYSTEM FRAMEWORK}

The PEMS consists of the head-mounted display (HMD), the drone, the mobile agent, the information server as illustrated in Fig. 1.

A HMD which may be a pair of smart glasses or an intelligent helmet is used to observe the specific power equipment usually. For the big and tall equipment that can't be watched from ground, a video drone is helpful to observe it from air. The video drone transmits real-time video to the HMD. Based on the information from mobile agent, HMD analyses the video, captures the outline of the equipment, selects one by the order of the maintenance staff and then superimposes needed data on the video image of the selected equipment. The business of the equipment is handled by data glove, the control key, gesture, voice, eye tracking, analog touch etc. 


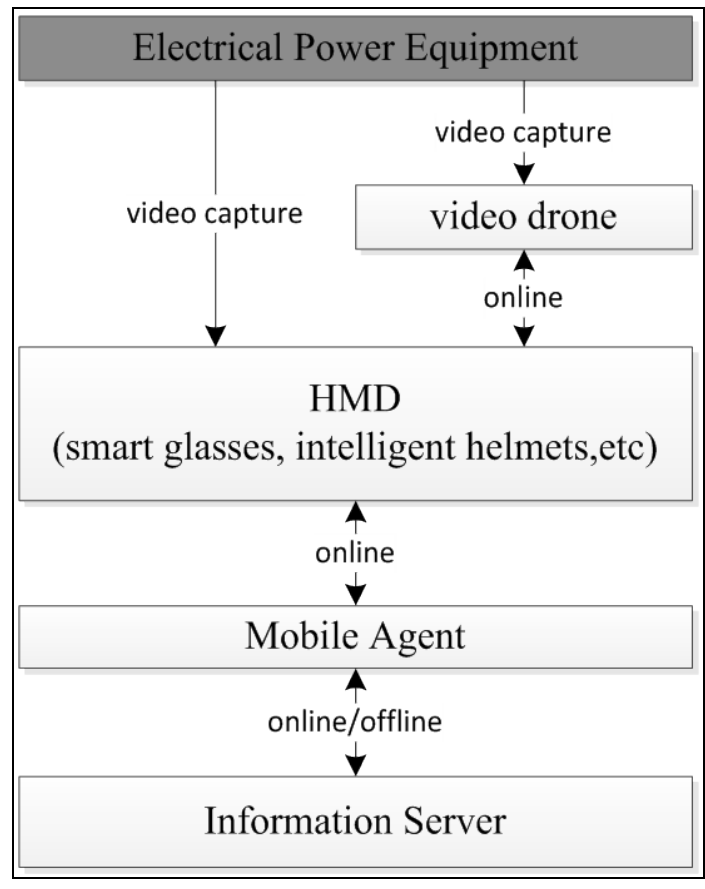

Figure 1. System composition.

Mobile agent software can be deployed in a mobile phone or a special mobile terminal. There is not too much limits to the shape of it. But it should be as small as possible for conveniently carried out. And it has basic network communication function. Usually, a SIM card is installed in it. When the on-site network performance is good enough it can exchange online data with information server; otherwise it can use the offline data which is downloaded in advance. The communication between HMD and mobile agent is fast and reliable.

We don't think of installing SIM card into HMD, though that is feasible in technology and can simplify the communication between HMD and information server. The mobile agent pattern is more efficient and adaptive.

Several equipment maintenance systems have been developed using AR techniques [3-7]. PEMS has some new key technologies contrast to them, which make it more applicable to equipment maintenance in the field.

\section{KEY TECHNOLOGIES}

\section{A. Image Recognition}

Image recognition is the most fundamental problem in AR. In PEMS, we present a similarity measure of matrices, and establish a similar discriminant function (SDF) of images. Based on the SDF, a novel feature extraction method is proposed for image recognition. For each class of equipment images, we calculate an optimal projection axis which maximizes the similarity among these equipment images for the class. Unlike the common methods of image recognition, a projective feature vector for a set of equipment image is extracted by projecting the image on the optimal projection axis of the class itself. Finally, we design a hierarchical classifier in the optimal discriminant space to recognize images.

Algebraic theory tells us that any real non-negative definite matrix can be transformed into a diagonal matrix by means of the orthogonal transformation. So can any matrix $\mathrm{F} \in \mathrm{R}^{\mathrm{mxn}}$ by means of the singular value decomposition. $\mathrm{F}$ has a characteristic matrix $\mathrm{T}_{\mathrm{k}}$ and a n-order non-negative reals $K_{\mathrm{k}}, \mathrm{k}=1,2,3, \ldots . \mathrm{n}$, if $\mathrm{F}$ is a non-negative definite matrix as in (1).

$$
\mathrm{F}=\sum_{k=1}^{n} \Lambda_{k} T_{k}
$$

$\mathrm{F}_{1}, \mathrm{~F}_{2}, \mathrm{~F}_{3} \ldots \mathrm{F}_{\mathrm{q}},(\mathrm{q}>1)$ are $\mathrm{m} \times \mathrm{n}$ order image matrices. A real $\mathrm{D}\left(\mathrm{F}_{1}, \mathrm{~F}_{2}, \mathrm{~F}_{3} \ldots, \mathrm{F}_{\mathrm{q}}\right)$ is defined as in (2).

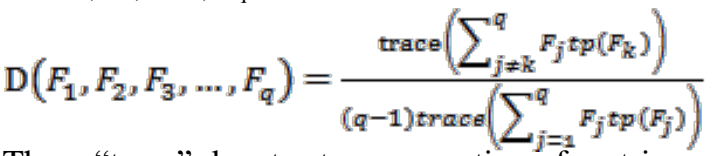

There "trace" denotes trace operation of matrix and "tp" denotes transpose. Then we call $\mathrm{D}\left(\mathrm{F}_{1}, \mathrm{~F}_{2}, \mathrm{~F}_{3 \ldots}, \mathrm{F}_{\mathrm{q}}\right)$ the similarity measure of matrices $\mathrm{F}_{1}, \mathrm{~F}_{2}, \mathrm{~F}_{3} \ldots, \mathrm{F}_{\mathrm{q}}$. There are two $150 \times 150$ equipment images $F_{1}, F_{2}$ shown in Fig. 2. D $\left(F_{1}, F_{2}\right)$ $=0.964$. A kind of similar relation among $F_{1}, F_{2}, F_{3} \ldots, F_{q}$ is described by $\mathrm{D}\left(\mathrm{F}_{1}, \mathrm{~F}_{2}, \mathrm{~F}_{3} \ldots, \mathrm{F}_{\mathrm{q}}\right)$. According to (1) and (2) we can prove that if $\mathrm{D}\left(\mathrm{F}_{1}, \mathrm{~F}_{2}, \mathrm{~F}_{3} \ldots, \mathrm{F}_{\mathrm{q}}\right)$ is nearly one, then the difference among $F_{1}, F_{2}, F_{3 \ldots}, F_{q}$ approaches zero.
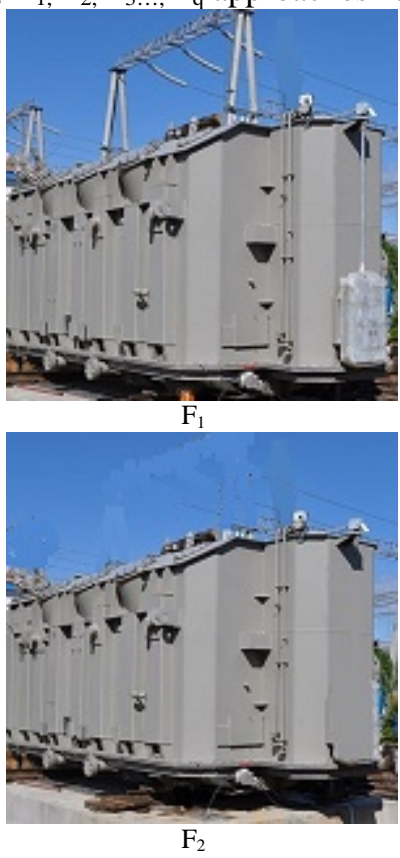

Figure 2. Two images F1 and F2 for the same transformer.

\section{B. Registration}

Registration is one of the most critical technologies in AR system. Registration is actually the process of aligning the computer-generated virtual objects with objects in the real world. Registration first determines the relationship between the virtual object and the observer, and then casts the virtual objects shadow into the viewer's vision through 
the correct geometric projection. Registration is generally divided into two classes, dynamic one and static one. Dynamic registration is the process of determining the relative position when there is a relative motion between the camera and the real object; static one is the process of doing it only if there is not any relative motion between them. In the current AR systems [8-10], the vast majority is the use of dynamic registration. Dynamic registration technology can be divided into two classes, those based on the tracker and those based on visual perception. In PEMS, We used an improved KLT algorithm to reduce the correlation with the illumination condition, and to ensure that the tracking will not fail when the maintenance staffs or the drones are moving fast. What they saw in the field experiment was like Fig. 3.

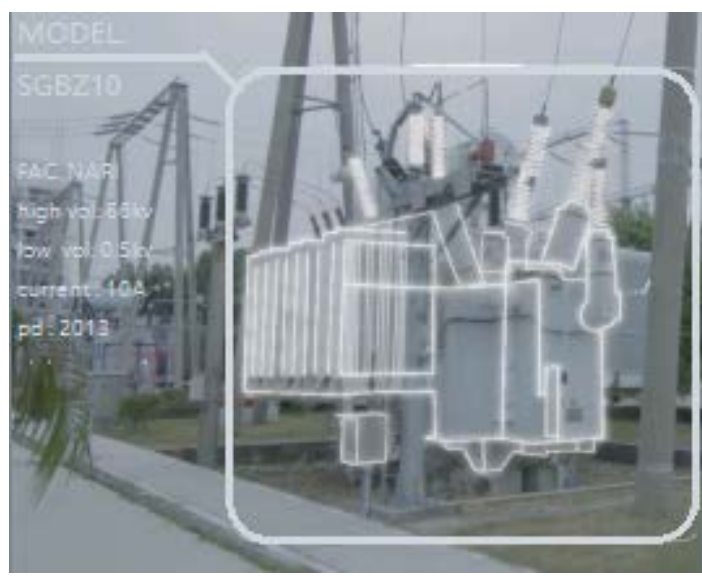

Figure 3. The real experiment scene.

\section{Mobile Agent}

IntelliSense is very important for electrical power equipment maintenance. The sensor technology can realize online real-time monitoring of transmission line operation and the state of the environment mainly includes the micro meteorological environment, icing, wire sag, temperature of conductor, conductor Aeolian vibration and galloping, line wind partial, site contamination degree, tower inclination, and monitoring are still expanding. AR has been becoming a new important technical method to realize the maintenance. If AR can be combined with those sensor technologies, the maintenance will be more accurate and efficient. However, there are many special characteristics in the transmission equipment maintenance, such as the on-site power supply difficulties, bad working environment, and communication blind areas. And there are many company's HMDs that can be selected. We don't know which company's product will dominate the future. PEMS is using mobile agent pattern to face these questions which is illustrated in Fig. 4. And the mobile agent can be the remote-control gadget of HMD in the time of need.

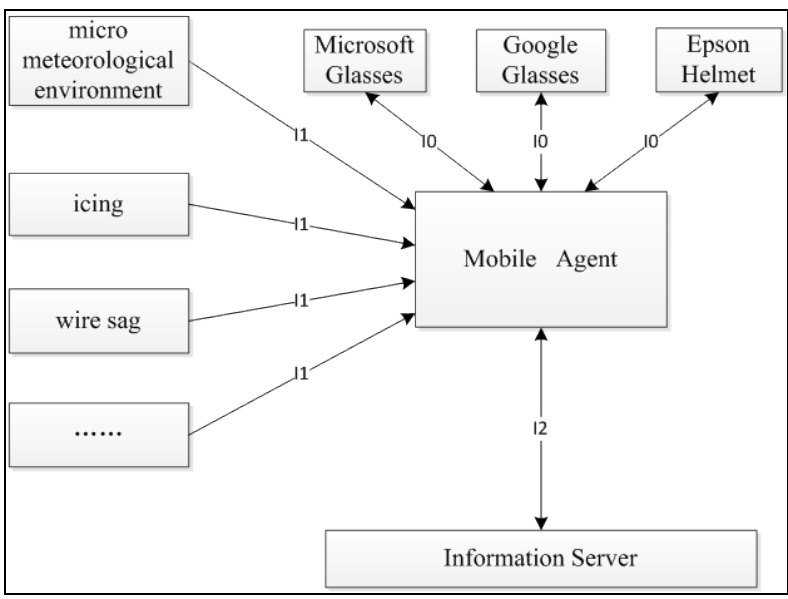

Figure 4. Mobile agent.

The communication protocol interface between the mobile agent and HMD is named I0. The one between the mobile agent and other sensors is named I1. The one between the mobile agent and information is named I2. When a new company HMD is used, we only need to add an I0; when a new sensor is met, we only need to add an I1. The I2 needs not to be edited when the HMD or the sensor is changed, which maybe influence the stability of the information system. I0, I1, I2 are separate.

\section{SUMMARY}

In order to test the efficiency of PEMS, it was used to recognize lots of electrical power equipment and help maintenance staffs handle business, just like Fig. 5. Experimental results have shown that PEMS has a good recognition performance, and can greatly reduce the workload of the maintenance staffs and improve work efficiency.

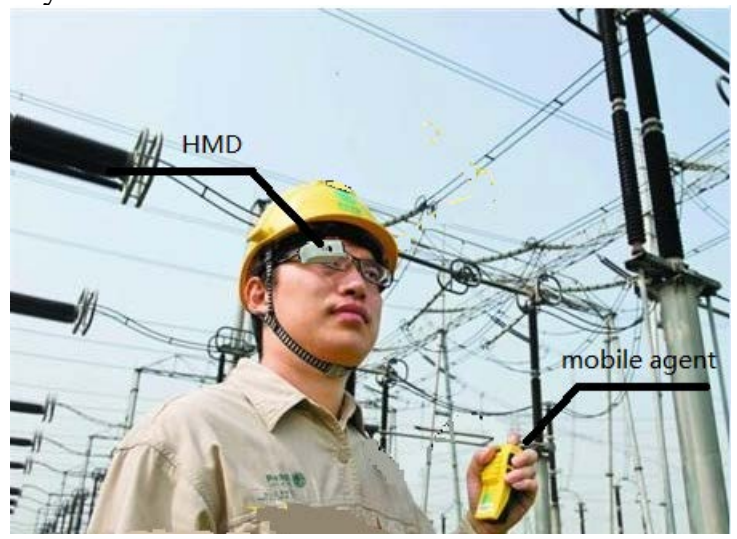

Figure 5. Work site

PEMS has the following advantages over other systems:

- It has a special image recognition algorithm for the electrical power equipment, which can effectively identify equipment when the equipment's appearance has changed by time or light.

- It can quickly capture equipment, and to "augment" the equipment. It's especially useful when the 
observer is moving fast, for example in a car or using a maintenance drone.

- It provides the drone to help the staff observe the equipment from air.

- It is more efficient and adaptive by using mobile agent pattern.

The application of augmented reality to electrical power equipment maintenance in this paper, not only can raise the power inspection work to a new level, but also can promote the power company's other business areas to upgrade. Through the integration of the power grid business, AR will change the mode of production, operation and management to a large extent, it will extend and expand the human sensory function, and bring new working mode. It can be predicted that with the AR in the power operation of the utility, the company will bring a new round of management innovation.

The application can also upgrade other software products for business function and display effect, improve the user experience of software products.

The application of AR is also facing many technical problems, and has a long way to go in the commercialization of products. Such as user in the line of sight of the moving image of the virtual real-time rendering problems, virtual image only in precision and integration of real-time and realistic environment under the premise can play a role, but these two requirements achieve powerful computing ability and memory space needs for support. And AR should be mobile. That means impossible for the user to carry bulky "super computer". Therefore, how to miniaturize hardware equipment, but also have powerful computation ability, memory space, long life, fast heat dissipation, stable network, and visualization reciprocal performance is AR equipment development direction.

AR application prospects are broad, can be determined in the near future, AR will appear in every corner of our life.

\section{REFERENCES}

[1] CIGRE WG C1-1, Asset management of transmission systems and associated CIGRE activities[R]. Paris, France, 2006.

[2] J.J. Smit, Trends in emerging technologies in power systems[C], International Conference on Future Power Systems, Amsterdam, Netherlands,2005, pp.1-7.

[3] T. Haritos, N.D. Macchiarella, A mobile application of augmented reality for aerospace maintenance training[C], Digital Avionics Systems Conference, 2005. IEEE, 2005:5.B.3-5.1-9 Vol. 1.

[4] S.K. Ong, J. Zhu, A novel maintenance system for equipment serviceability improvement [J], CIRP Annals - Manufacturing Technology, 2013, 62(1):39-42.

[5] M. Kleiber, T. Alexander, C. Winkelholz, et al., User-centered design and evaluation of an integrated AR-VR system for telemaintenance[C], IEEE International Conference on Systems, Man, and Cybernetics. IEEE, 2012: 1443 - 1448.

[6] S. Benbelkacem, N. Zenati-Henda, Belhocine, et al., Augmented Reality Platform for Solar Systems Maintenance Assistance [J]. 2010.

[7] H. Martínez, S. Laukkanen, J. Mattila, A New Hybrid Approach for Augmented Reality Maintenance in Scientific Facilities [J]. International Journal of Advanced Robotic Systems, 2013, 10(321):418-429.

[8] M. Horvat, Rendering of dynamic objects in Augmented reality [J]. Hrvatska znanstvena bibliografija i MZOS-Svibor, 2013.

[9] M. Tatzgern, D. Kalkofen, D. Schmalstieg, Dynamic compact visualizations for augmented reality [J]. 2013:3-6.

[10] N. Lelos, P. Campos, K. Sugand, et al., Augmented reality dynamic holography for neurology [J]. Journal of Neurology Neurosurgery \& Psychiatry, 2014, 85(10). 